\title{
6in4 Tunnel Based IPv6 Transition Solution for IPv4 Mobile Terminals
}

\author{
Jingwen Gao and Qin Zhao
}

\begin{abstract}
Along with the commercialization of IPv6 in global scale, the whole IPv6 industry chain will mature gradually. However, even after a real world deployment of IPv6, a huge amount of legacy IPv4 devices and applications won't disappear just overnight. Despite the solutions and tools developed to help ease the IPv4/IPv6 transition, few of them is focused on dealing with the situation of mobile terminals which may move among different WIFI and 3G networks. This paper documents a novel 6in4 tunnel based transition solution for IPv4 mobile terminals to access to IPv6 resources, together with the prototype implementation and evaluation. The aim of this paper is to provide solution for IPv4 mobile terminals, which grow in quantity dramatically day by day, to peacefully migrate to IPv6.
\end{abstract}

Index Terms - ISATAP, mobile terminal, transition, tunnel.

\section{INTRODUCTION}

As smart mobile terminals, including smart phones, tablet PCs, and other entertainment platforms, etc., becoming increasingly attractive, not a day will go without using these handy little things for daily social or official routines. The user group of smart mobile devices grows dramatically in size in recent years, as with the frequency the users searching the Internet with these devices. Mobile devices provide people with handy access to the Internet. One thing to note is that mobile device requires a globally-unique IP address to stay online, which is hardly possible as the number of mobile devices overwhelms that of the IPv4 addresses.

Since the IPv6 was raised as a solution to the IPv4 address exhaustion in the middle of 1990s, many years have passed by, researchers and developers paying uncountable efforts to the design and implementation of IPv6. IPv6 provides 296 times larger address space than its predecessor, with many other noticeable advantages. IPv6 is believed to finally replace the position where IPv4 currently stands. The migration to IPv6 from IPv4 in mobile networks has gained much attention from all walks of life these years. However, a good amount of time is needed before we can actually step into an IPv6-only world. For mobile terminals to reach to the Internet via IPv6 as convenient as they can now via IPv4, we need support from both the mobile network and mobile terminals themselves.

In terms of mobile network, in the standardization of IPv6, the organization IETF undertakes the responsibility of the completion of IPv6 protocols, while 3GPP assume

Manuscript received March 20, 2014; revised June 28, 2014. This work was supported in part by the CNGI project "Research and Trial on Evolving Next Generation Network Intelligence Capability Enhancement (NICE)".

The authors are with the Institute of Network Technology, Beijing University of Posts and Telecommunications, Beijing 100876, China (e-mail gaojingwen@bupt.edu.cn, zhaoqin@bupt.edu.cn). responsibility for the application of IPv6 in 3G core network. Up till now, the IPv6 migration strategy for $3 \mathrm{G}$ core network is almost ready. Several standards have been proposed in 3GPP, while more work is required for the whole mobile network to transfer to IPv6 [1]. Mobile devices are incapable to reach IPv6 through mobile network now and would probably not be able to in near future.

In terms of mobile terminals, most devices connect to the Internet via either WIFI or 3G. There's no obstacle in the hardware level for mobile devices to access to IPv6 via WIFI. The only thing that is required is the operating system supports IPv6, which has been satisfied by major mobile operating systems such as Android and IOS. Yet things become a little different for 3G. IPv4 and IPv6 cannot communicate with each other directly by design. Once a mobile device tries to access to IPv6 via 3G, assume the mobile network supports IPv6, the device itself needs to be configured both in the operating system and the hardware (mainly the chip) with the corresponding capabilities. In the respect of hardware, few handsets support IPv6 in its chip. Manufacturers lack the momentum to produce such terminals because IPv6, although with many advantages, offers no new service or better user experience over IPv4.

IPv4 still carries the vast majority of Internet traffic now. The migration from IPv4 to IPv6 would last for the coming ten to fifteen years, as stated in [2]. In such long period of transition, it is of great significance to provide solutions to accessing IPv6 for IPv4 mobile terminals.

In summary, there is still a long way to go before the full deployment of IPv6. It should be given good consideration to provide convenience for IPv4 mobile terminals to access to IPv6. That is the motivation of the solution to be introduced in this paper.

\section{TRANSITION MECHANISMS AND MOBILITY CONSIDERATION}

\section{A. Transition Mechanisms}

Before IPv6 completely supplants IPv4, transition mechanisms are needed for IPv4/IPv6 interoperability. Commonly, there are three categories of transition strategies: dual stack, translation, and tunneling.

In dual stack strategy we configure devices with the ability to run IPv4 and IPv6 in parallel. It allows hosts to reach contents of both protocols simultaneously, and provide flexibility for applications that run over IPv4 and/or IPv6. Dual stack allows organizations to traverse from IPv4 to IPv6 gradually. Besides, it is supported by all major operating systems up till now. That is why dual stack strategy is 
recommended to most networks for the migration. For mobile terminals, especially, there is a gap before dual stack devices can be deployed for extensive commercialization, as has stated in the Introduction part of this paper.

The translation strategy translates packets from one IP version to the other. A translator is involved in this process, which is either the client device itself or other device in the network. The translated packets then can be transmitted over the network and finally be understood by the destination device. Possibly the translation process is placed on a network device close to the client device. This network device, named the translator, however, becomes the bottleneck of this strategy. For packets which use embedded IP addresses, special algorithms are needed for effective translation for each separate application protocol. Furthermore, the translator needs to track the client's IP address for translation, while the IP address of mobile terminal changes from time to time. While taking mobility of client devices into account, translation becomes far from cost-effective.

Another popular transition technique, tunneling, encapsulates IPv6 packets into IPv4 packets, using IPv4 as link layer of IPv6 (or vice versa). One of the most commonly used tunnel based mechanisms is ISATAP [3]. ISATAP (Intra-Site Automatic Tunnel Addressing Protocol) is a kind of 6in4 tunneling strategy, which encapsulates IPv6 in IPv4 packet for transmission over IPv4 only network. The 'protocol' field of the IPv4 header is set to value 41 (IPPROTO_IPV6) to indicate 6in4 [4] tunneling packets. With increasingly wider application of ISATAP, it has been supported by most platforms. Android devices are capable to use their built-in SIT module for establishing ISATAP tunnels. This is a huge benefit of ISATAP while other tunneling techniques, such as 6rd [5], have no supporting built-in modules from the operating system itself. It provides a possible way for mobile terminals to access to IPv6 in the transition period. Moreover, after careful and comprehensive tests, the SIT module has been proved to be supported by all Android platform devices tested, in the kernel level. This is the basis of the solution proposed in this paper.

\section{B. Mobility Consideration}

Mobile terminals reach the Internet via wireless or mobile network. Generally, mobile users travel from place to place, moving from one network to another, either WIFI or 3G. This causes the IP addresses of mobile terminals to change from time to time, making certain transition strategies like translation to present poor performance.

What's more, the overhead that transition techniques add to realize IPv6 access should also be taken into account. For tunneling techniques like OpenVPN [6], IPv6 data must be encapsulated several times before it can be transmitted to destination, which burdens substantial additional overhead in management and data traffic aspects.

Hence, the ISATAP tunneling strategy is most suitable for mobile terminals migrating to IPv6, for the reason that it is naturally supported by the major smart phone platform Android in the kernel level, and its efficiency in encapsulating IPv6 packets. Adaptations are needed before ISATAP tunnels can be utilized by mobile users, as well as mobility considerations.

\section{6IN4 TUNNEL BASED IPv6 TRANSITION SOLUTION}

\section{A. ISATAP}

The ISATAP protocol defines a method to generate a link-local IPv6 address from an IPv4 address, and a mechanism to perform neighbor discovery on top of IPv4. The 128-bit link-local IPv6 address of the client is consisted of a 64-bit IPv6 address prefix, which is obtained from the ISATAP server, a 32-bit identifier assigned by IANA, and the client's original 32-bit IPv4 address, as shown in Table I below. The construction of this IPv6 address can be automated or manually configured. After this construction, the host becomes an ISATAP client. The embedded IPv4 address could be either public or private, which saves public IPv4 address resources.

When one ISATAP client is about to communicate with another ISATAP client in the same IPv4 domain, the embedded IPv4 addresses will be subtracted from the IPv6 addresses to determine the tunnel's source and destination IPv4 addresses. Then the encapsulated packet can be transmitted through the IPv4 network. No modification is required on the current IPv4 network infrastructure.

ISATAP not only enables the communication between two ISATAP clients as described above, but also provides support for the communication between IPv4 hosts and other IPv6 devices of separate domain. The 6in 4 packets are relayed by the ISATAP server between the IPv4 ISATAP client and its IPv6 peer. One limitation is that, the ISATAP client and the ISATAP server are required to be located in one IPv4 domain, or the ISATAP client should possess a public IPv4 address.

Despite the fact that mobile network scenario was within the domain of applicability of ISATAP when the protocol was proposed [3], little research or development has been found towards the application of ISATAP in this scenario. Here substantial investigations and tests have been done towards the applicability of ISATAP tunnels in the mobile network scenario. Moreover, adaptations and improvements have been conducted to make it suitable for existing network conditions and mobile terminals.

TABLE I: ISATAP IPV6 ADDRESS COMPOSITION

\begin{tabular}{|c|c|c|}
\hline 2001:db8:: & 0:5EFE: & x.x.x.x \\
\hline 64-bit IPv6 prefix & $\begin{array}{c}\text { 32-bit } \\
\text { identifier }\end{array}$ & $\begin{array}{c}\text { 32-bit } \\
\text { embedded } \\
\text { IPv4 address }\end{array}$ \\
\hline
\end{tabular}

\section{B. Gin4 Tunnel Based IPv6 Transition Solution}

The 6in4 tunnel based IPv6 transition solution for IPv4 mobile terminals proposed in this paper is an application of the ISATAP protocol in the scenario of mobile terminals.

As have stated before, tests have been conducted towards the ability of establishing ISATAP tunnels with SIT module on major smart mobile devices, mainly on the Android platform. Tested devices include popular models of mainstream handset manufacturers, with results listed below in Table II. This is the fact backing the adoption of ISATAP in the proposed transition solution for mobile terminals.

As shown in Fig. 1 below, the mobile terminal is originally within the domain of IPv4 mobile network $A$. Here in this 
solution, a client software application named 6able has been designed and implemented for mobile terminals. Mobile terminals use the 6able application to establish ISATAP tunnels with the ISATAP server. After the tunnel has been established, the dual stack ISATAP server works as a relay in communication.

After a certain period of time, the mobile terminal moves to another IPv4 mobile network $B$. Its IPv4 address changes after the handoff. Now the current ISATAP tunnel becomes invalid. To handle this problem, the moment a handoff event is captured, the 6able application detects the IPv4 address change, and reconfigures the ISATAP tunnel.

One thing to note is that, different with normal ISATAP client applications compliant with the protocol, the 6able application is implemented on the basis of the built-in SIT module of Android devices. This means the communication between two 6able clients has to be relayed by the ISATAP server, even when they are within the same site.

TABLE II: MOBILE TERMinALS’ ABILITY OF ESTABLISHING ISATAP WITH SIT MODULE

\begin{tabular}{|l|l|l|l|}
\hline Manufacturers & Platform & $\begin{array}{l}\text { Num. of } \\
\text { models } \\
\text { tested }\end{array}$ & Result \\
\hline SAMSUNG & Android 4.0,4.2 & 5 & Support \\
\hline LG & Android 4.0 & 2 & Support \\
\hline HUAWEI & Android 4.0 & 2 & Support \\
\hline Others & Android 4.0 & 2 & Support \\
\hline
\end{tabular}

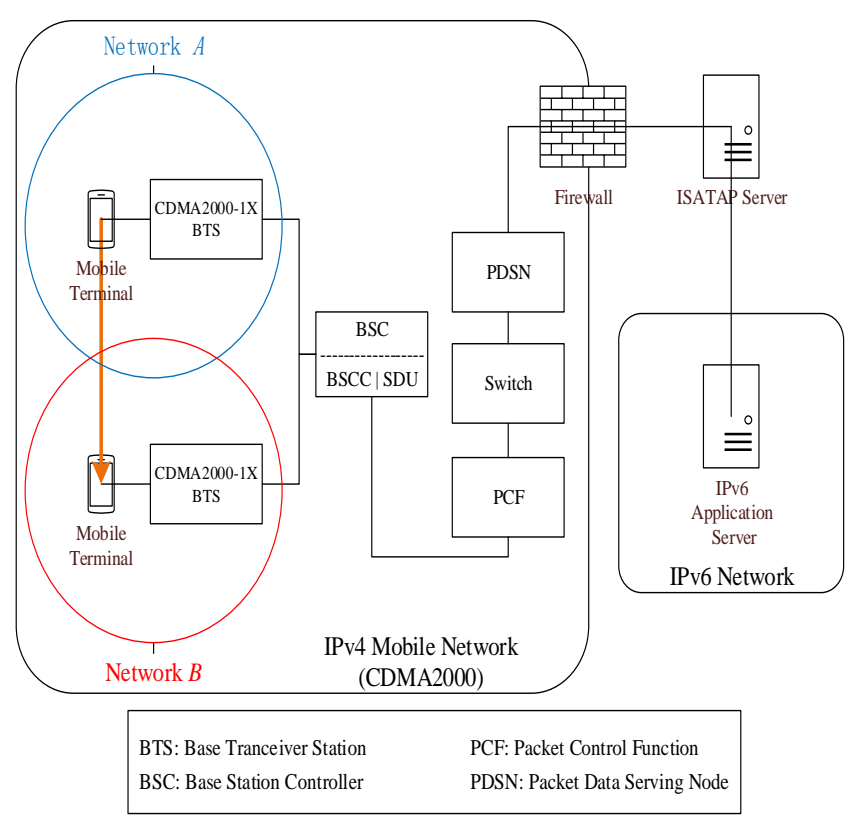

Fig. 1. ISATAP in mobile network scenario.

\section{PROTOTYPE IMPLEMENTATION}

A working prototype of the 6in 4 tunnel based transition solution has been implemented and has for several months been undergoing field trials within the Network Information
Center of Beijing University of Posts and Telecommunications. The implementation is based on the Android platform, and has been tested thoroughly on devices of Android version 4.0 and beyond. The client software, namely 6able, designed and implemented in this prototype is written in Java, and a Graphical User Interface is provided for operation.

The main procedure of establishing/reestablishing ISATAP tunnel can be outlined with the following Fig. 2, which is the key functionality behind the 6able application. As the figure shows, when first establishes a tunnel, the 6able application would at first obtain the IPv4 address of the host, and then check if the address is suitable for establishing tunnel with the given ISATAP server. If the address is qualified, then a tunnel is established with several demands which will set the attributes of the tunnel with the help of the SIT module of the Linux kernel. Otherwise, a prompt is presented to the user. After that, user interactions data is collected, with the Google Analytics [7] tool. Besides, the application would always be listening on handoff events. Once the mobile device moves from one network to another, the application would be informed of the handoff, and then silently configure the tunnel again with the new IPv4 address. The actions handling handoffs are apparent to the user.

The whole solution needs no modification of the existing IPv4 mobile network. IPv4 mobile terminals can access to IPv6 resources, with mobility issues taken into account. The prototype implementation was completed in around two man months from design to implementation, with approximately 4,000 lines of code of the 6able client application. The tests and data collection are completed in around 5 man months from September 2013 to January 2014.

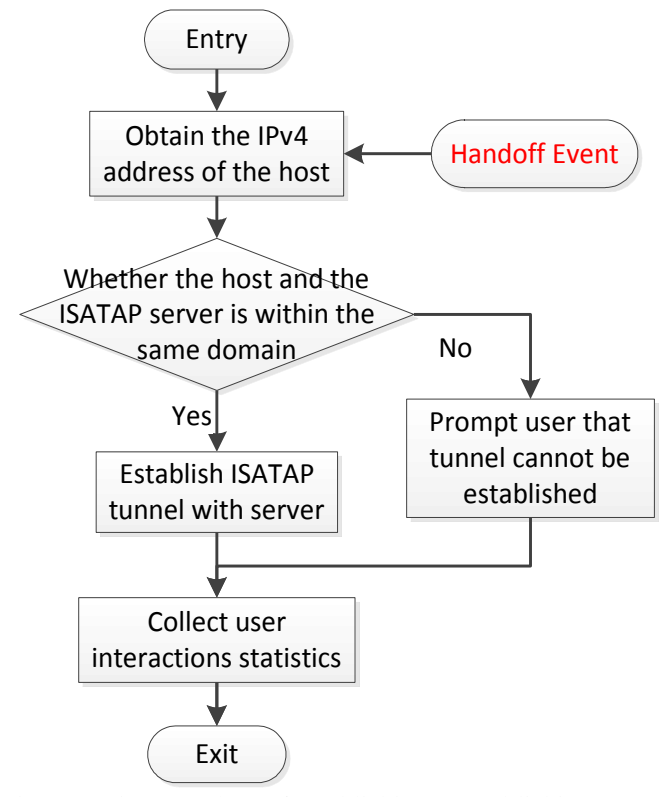

Fig. 2. Main procedure of establishing/reestablishing tunnel.

\section{Evaluation}

Since the completion of implementation of the client application, 6able has been undergoing sufficient tests and trials. Firstly, the application has been tested through 15 test cases. Secondly, it has been spread to a number of selected 
users for field trials.

The environmental configuration is consisted of an Android handset (Model C8812 of HUAWEI the manufacturer), a dual-stack ISATAP server configured with public IPv4 address, IPv4-only network (the exiting 3G network of China Telecom), and several IPv6-only online sites for testing (including ipv6.google.com etc.). A test is successful when the user could reach the IPv6-only sites after the tunnel is established.

For the tens of hundreds of tests that we have conducted towards the application, test results have proven that the application manages to reach its aim and the solution proposed in this paper is practical. Fig. 3 below shows an example of successfully establishing the tunnel, and the host managed to get its IPv6 address. The IPv6 address shown can be seen to be constructed with the IPv4 address. After that, user could open links to IPv6-only sites as they wish.

As have mentioned before, user interactions data has been collected with the Google Analytics tool for the past several months. Here are the results gathered from Google Analytics in Fig. 4 and Table III below. Fig. 4 shows the number of sessions generated per month from the 6able application, whereas Table III is a list of statistics, including number of session, number of screen view, number of screen view per session, and average session duration, against different languages of the source devices.

TABLE II: USER INTERACTIONS STATISTICS FROM SEP. 2013 TO JAN. 2014

\begin{tabular}{|c|l|l|l|}
\hline Language & zh-cn & en-us & Total \\
\hline Session & $\begin{array}{l}1,067 \\
(99.63 \%)\end{array}$ & $\begin{array}{l}4 \\
(0.37 \%)\end{array}$ & 1,071 \\
\hline Screen View & $\begin{array}{l}1,475 \\
(99.73 \%)\end{array}$ & $\begin{array}{l}4 \\
(0.27 \%)\end{array}$ & 1,479 \\
\hline $\begin{array}{c}\text { Screen View per } \\
\text { Session }\end{array}$ & 1.38 & 1.00 & 1.38 \\
\hline $\begin{array}{c}\text { Average Session } \\
\text { Duration }\end{array}$ & $00: 00: 45$ & $00: 00: 00$ & $00: 00: 45$ \\
\hline
\end{tabular}

The results from all the tests and trials have shown the practical significance and effectiveness of the proposed solution in the respective of providing IPv6 access to IPv4 mobile terminals, without any hardware or software modifications on the current IPv4 network infrastructure.

\section{Welcome to IPv6!}

OFF

IPV6 address: 2001:da8:202:107:0:5efe:73aa:b4b

IPV4 address: 115.170.11.75

DNS address: 219.141 .136 .10

Fig. 3. 6able application screenshot.

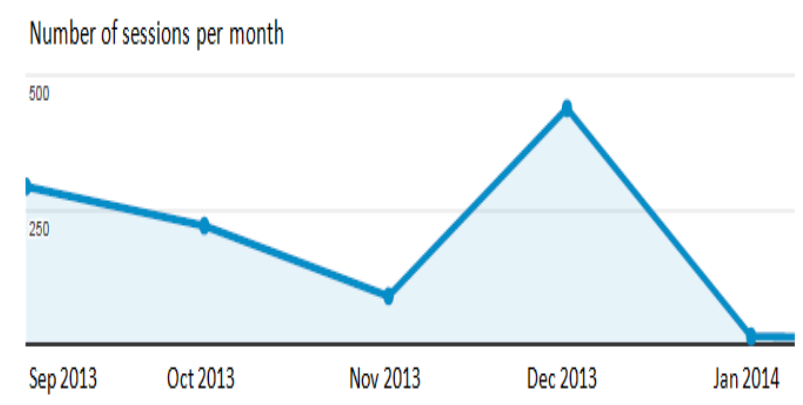

Fig. 4. User interactions statistics from Sep. 2013 to Jan. 2014

\section{CONCLUSIONS}

This paper has proposed a 6in4 tunnel based IPv6 transition solution for IPv4 mobile terminals, so that they can easily get access to IPv6. The solution proposed could also provide possible solution for telecom carriers who are eager to offer IPv6 services without much improvements or replacements done on their current system. It is unique for the fact that it aims at providing a suitable way for existing mobile terminals to migrate from IPv4 to IPv6. With the application software designed and implemented, IPv4 mobile terminals are able to reach IPv6 resources in the experimental environment. Moreover, host mobility has been taken into account, and mobile terminals can "stay on IPv6" when they move from one network to another, as long as the new IPv4 address assigned to them allows a new tunnel to be established. Qualitative and quantitative tests have proved the practical significance and effectiveness of the solution.

Further work in this area would probably involve three aspects. First, the client application could be improved to determine whether its communication peer is in the same site. By implementing this functionality, the traffic through the ISATAP server could be reduced. Meanwhile, communication efficiency will be improved in this case. Second, more ISATAP servers can be deployed for larger scale of tests. By doing this, it is also expected to share the load on the only server. That is what we are now actively making efforts for its realization. Third, we are planning to design and implement a kind of NAT device that is aware of 6in4 packets. The NAT device would do translations of addresses not only to the IPv4 header of 6in4 packet, but also the inner IPv6 header. By adding this to the experimental environment, the mobile terminal is expected to be able to reach to IPv6, even when the ISATAP server is in different IPv4 domain with the mobile terminal.

\section{ACKNOWLEDGMENT}

This paper is supported by the CNGI project "Research and Trial on Evolving Next Generation Network Intelligence Capability Enhancement (NICE)".

\section{REFERENCES}

[1] B. Yan, C. Zhang, X. Tian, and L. Jiang, "Analysis of the research and development of key techniques of IPv6 terminals," Information and Communications Technologies, vol. 3, pp. 50-53, 2013.

[2] X. Li, "Panic: IPv6," China Computer World, vol. 15, pp. 20-23, April 2012.

[3] F. Templin, T. Gleeson, and D. Thaler. "Intra-site automatic tunnel addressing protocol (ISATAP),” IETF RFC 5214, March 2008 
[4] R. Gilligan and E. Nordmark, "Basic transition mechanisms for IPv6 hosts and routers," IETF RFC 4213, October 2005.

[5] R. Despres, "IPv6 rapid deployment on IPv4 infrastructures (6rd)," IETF RFC 5569, January 2010.

[6] OpenVPN. Open Source VPN. [Online]. Available: http://openvpn.net/ t/

[Online]. Available: https://developers.google.com/analytics/

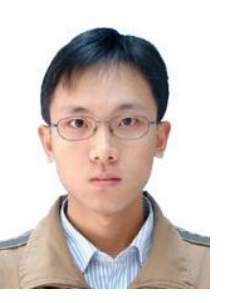

Qin Zhao received the B.S. degree in BUPT and he works as an engineer in BUPT. His research interests include IPv6 transition technologies and next generation Internet architecture.

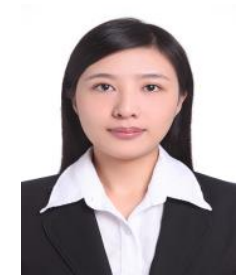

Jingwen Gao received the B.S. degree from Beijing University of Posts and Telecommunication (BUPT) and Queen Mary, University of London (QMUL) in 2012, in telecommunications engineering with management. She is currently pursuing the M.S degree with the Institute of Network Technology, BUPT. Her research interests include IPv4/IPv6 transition technologies and mobile network. 